\title{
Changing the static and dynamic lattice effects for the improvement of the ionic transport properties within the argyrodite $\mathrm{Li}_{6} \mathrm{PS}_{5-\mathrm{x}} \mathrm{Se}_{\mathrm{x}} \mathrm{I}$
}

Roman Schlem ${ }^{\mathrm{a}, \mathrm{b}}$, Michael Ghidiu, ${ }^{\mathrm{a}, \mathrm{b}}$, Sean P. Culver ${ }^{\mathrm{a}, \mathrm{b}}$, Anna-Lena Hansen ${ }^{\mathrm{c}}$, Wolfgang G. Zeier*a,b

anstitute of Physical Chemistry, Justus-Liebig-University Giessen, Heinrich-Buff-Ring 17, D35392 Giessen, Germany.

${ }^{b}$ Center for Materials Research (LaMa), Justus-Liebig-University Giessen, Heinrich-Buff-Ring 16, D-35392 Giessen, Germany.

${ }^{c}$ Institute for Applied Materials - Energy Storage Systems, Karlsruhe Institute of Technology, Hermann-von-Helmholtz-Platz 1, D-76344 Eggenstein-Leopoldshafen, Germany

\section{Corresponding Authors}

*wolfgang.g.zeier@pc.jlug.de; 


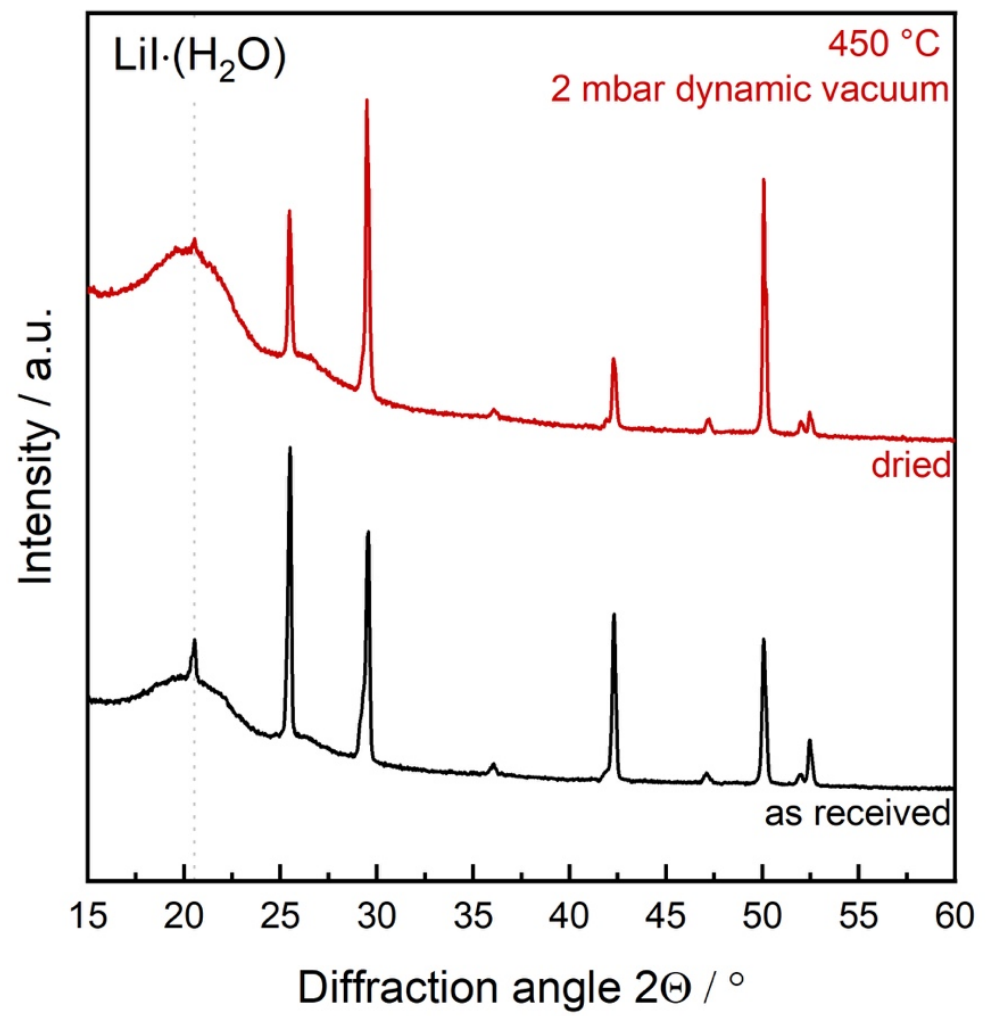

Figure S1: Lil precursor before and after a drying step. The most visible impurity reflection in the synthesized compounds is highlighted with a vertical dotted line. The drying reduced the amount of Lil $\cdot\left(\mathrm{H}_{2} \mathrm{O}\right)$ impurity phase, but even multiple drying steps did not result in a phase-pure precursor.

Table S1: Used constraints for the Rietveld-refinements against capillary X-Ray data. The full amount of substituted Selenium was set to the tetrahedral Wyckoff $16 e$ site reduced by the occupancies on the remaining possible sites.

\begin{tabular}{|c|c|c|c|c|c|c|}
\hline \multirow{2}{*}{ Atom } & \multirow{2}{*}{$\begin{array}{l}\text { Wyckoff } \\
\text { position }\end{array}$} & \multicolumn{3}{|c|}{ Atomic coordinates } & \multirow{2}{*}{ Occ. } & \multirow{2}{*}{$B_{i s o} / \AA^{2}$} \\
\hline & & $x$ & $y$ & $z$ & & \\
\hline S1 & $4 d$ & 0.25 & 0.25 & 0.25 & $1-(1-[1-(o c c 2+o c c 1)])-o c c 3$ & Varl \\
\hline Se1 & $4 d$ & 0.25 & 0.25 & 0.25 & occ3 & Varl \\
\hline I1 & $4 d$ & 0.25 & 0.25 & 0.25 & $1-[1-(o c c 2+o c c 1)]$ & Varl \\
\hline $\mathbf{I} 2$ & $4 a$ & 0 & 0 & 1 & $1-(o c c 2+o c c 1)$ & Var2 \\
\hline $\mathrm{Se} 2$ & $4 a$ & 0 & 0 & 1 & occ2 & Var2 \\
\hline S2 & $4 a$ & 0 & 0 & 1 & occl & Var2 \\
\hline S3 & $16 e$ & Pos 1 & $-P o s 1$ & $0.5+\operatorname{Pos} 1$ & $1-\left[\frac{\mathrm{x}}{4}-0.25 \cdot(\operatorname{occ} 2+\operatorname{occ} 3)\right]$ & Var3 \\
\hline Se3 & $16 e$ & Pos 1 & $-P o s 1$ & $0.5+\operatorname{Pos} 1$ & $\frac{\mathrm{X}}{4}-0.25 \cdot($ occ $2+o c c 3)$ & Var3 \\
\hline $\mathbf{P}$ & $4 b$ & 0 & 0 & 0.5 & 1 & Var4 \\
\hline Li1 & $24 g$ & 0.25 & 0.018 & 0.75 & 0.219 & 5 \\
\hline Li2 & $48 h$ & 0.2956 & 0.0266 & 0.7044 & 0.391 & 5 \\
\hline
\end{tabular}


Table S2: Rietveld-refinement data of $\mathrm{Li}_{6} \mathrm{PS}_{5} \mathrm{l}$ and corresponding fit-residuals.

\begin{tabular}{|c|c|c|c|c|c|c|}
\hline $\begin{array}{l}\mathrm{Li}_{6} \mathrm{PS} \\
\text { Lattice }\end{array}$ & meter: 10.1 & 1) & . & & & \\
\hline$R_{\mathrm{WP}}=5$ & $7, R_{\exp }=3.6$ & Goodness of & t: 1.63 & & & \\
\hline Total is & ity amount & 6 LiI & & & & \\
\hline Atom & Wyckoff & & iic coordina & & Occ. & $B_{\text {iso }} / \AA^{2}$ \\
\hline Atom & position & $x$ & $y$ & $z$ & Occ. & \\
\hline S1 & $4 d$ & 0.25 & 0.25 & 0.25 & $0.983(2)$ & $1.35(7)$ \\
\hline I1 & $4 d$ & 0.25 & 0.25 & 0.25 & $0.017(2)$ & $1.35(7)$ \\
\hline I2 & $4 a$ & 0 & 0 & 1 & $0.983(2)$ & $2.10(3)$ \\
\hline S2 & $4 a$ & 0 & 0 & 1 & $0.017(2)$ & $2.10(3)$ \\
\hline S3 & $16 e$ & $0.1162(1)$ & $-0.1162(1)$ & $0.6162(1)$ & 1 & $1.24(4)$ \\
\hline $\mathbf{P}$ & $4 b$ & 0 & 0 & 0.5 & 1 & $0.91(5)$ \\
\hline Li1 & $24 g$ & 0.25 & 0.018 & 0.75 & 0.219 & 5 \\
\hline Li2 & $48 h$ & 0.2956 & 0.0266 & 0.7044 & 0.391 & 5 \\
\hline
\end{tabular}




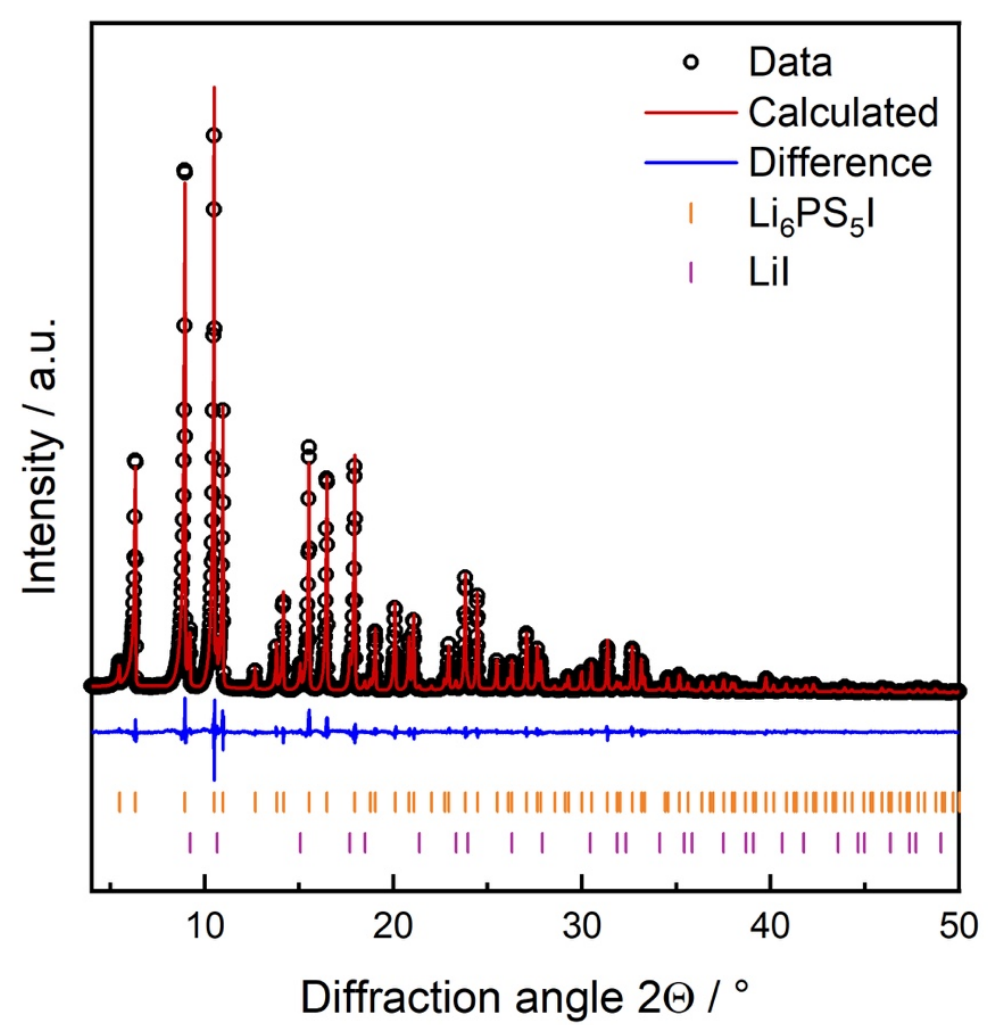

Figure S2: Diffraction pattern for $L_{6} P_{6} S_{5}$ ( (black dots), calculated structure using a Rietveld-refinement (red line) and difference curve (blue). Minor amounts of Lil impurities can be found.

Table S3: Rietveld-refinement data of $\mathrm{Li}_{6} \mathrm{PS}_{4} \mathrm{Se}_{1}$ l and corresponding fit-residuals.

\section{$\mathrm{Li}_{6} \mathrm{PS}_{4} \mathrm{Se}_{1} \mathrm{I}$}

Lattice Parameter: 10.2182(1)

$R_{\mathrm{WP}}=5.61 \%, R_{\mathrm{exp}}=5.27 \%$, Goodness of fit: 1.06

Total impurity amount: $3 \%\left(\mathrm{LiI}, \mathrm{Li}_{2}(\mathrm{~S} / \mathrm{Se})+\mathrm{LiI}\left(\mathrm{H}_{2} \mathrm{O}\right)\right.$

\begin{tabular}{ccccccc}
\hline \multirow{2}{*}{ Atom } & $\begin{array}{c}\text { Wyckoff } \\
\text { position }\end{array}$ & \multicolumn{3}{c}{ Atomic coordinates } & \multirow{2}{*}{ Occ. } & $\boldsymbol{B}_{\text {iso }} / \AA^{\mathbf{2}}$ \\
\cline { 3 - 6 } S1 & $4 d$ & 0.25 & 0.25 & 0.25 & $0.72(2)$ & $1.43(8)$ \\
\hline Se1 & $4 d$ & 0.25 & 0.25 & 0.25 & $0.25(2)$ & $1.43(8)$ \\
\hline $\mathbf{I 1}$ & $4 d$ & 0.25 & 0.25 & 0.25 & $0.03(1)$ & $1.43(8)$ \\
\hline $\mathbf{I} 2$ & $4 a$ & 0 & 0 & 1 & $0.97(1)$ & $2.39(5)$ \\
\hline Se2 & $4 a$ & 0 & 0 & 1 & $0.00(1)$ & $2.39(5)$ \\
\hline S2 & $4 a$ & 0 & 0 & 1 & $0.032(5)$ & $2.39(5)$ \\
\hline S3 & $16 e$ & $0.1176(1)$ & $-0.1176(1)$ & $0.6176(1)$ & $0.813(6)$ & $1.97(6)$ \\
\hline Se3 & $16 e$ & $0.1176(1)$ & $-0.1176(1)$ & $0.6176(1)$ & 1 & $1.97(6)$ \\
\hline $\mathbf{P}$ & $4 b$ & 0 & 0 & 0.5 & 0.219 & $1.35(9)$ \\
\hline $\mathbf{L i 1}$ & $24 g$ & 0.25 & 0.018 & 0.75 & 0.391 & 5 \\
\hline $\mathbf{L i} 2$ & $48 h$ & 0.2956 & 0.0266 & 0.7044 & 5 \\
\hline
\end{tabular}




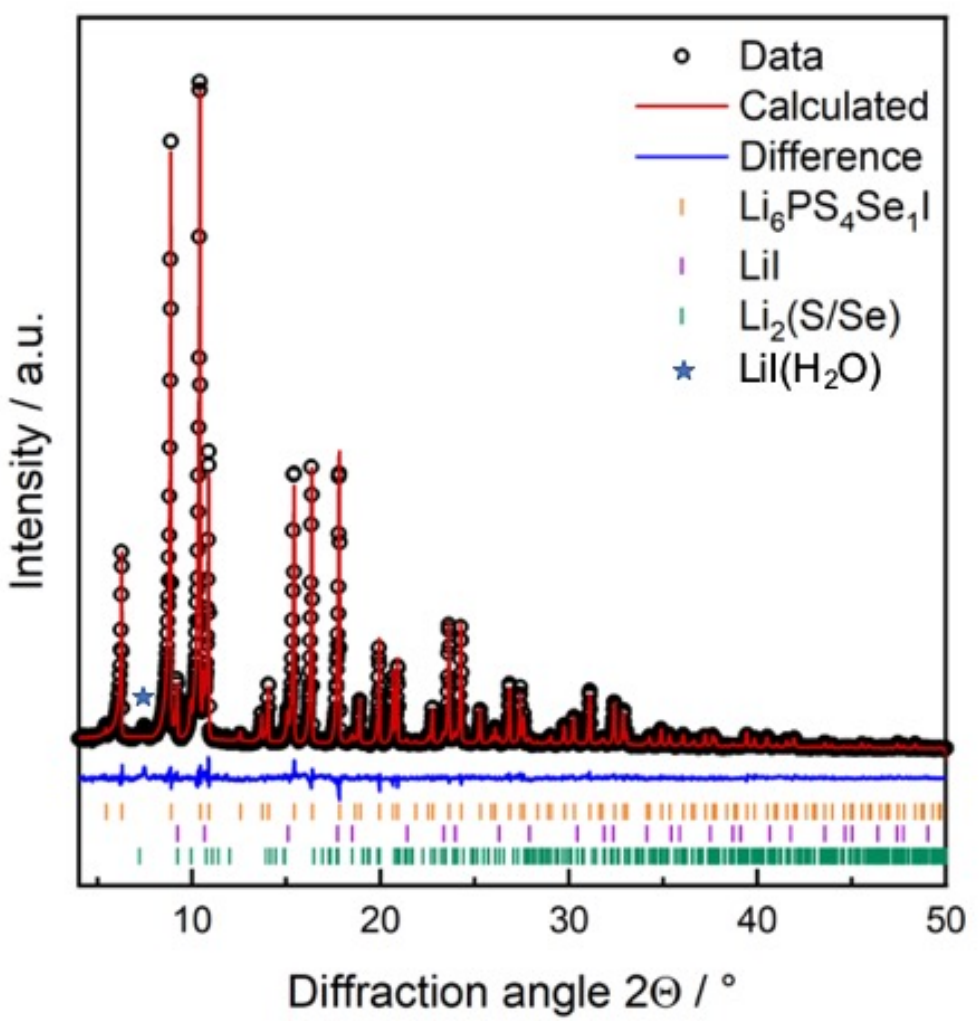

Figure S3: Diffraction pattern for $\mathrm{Li}_{6} \mathrm{PS}_{4} \mathrm{Se}_{1}$ l (black dots), calculated structure using a Rietveld-refinement (red line) and difference curve (blue). Minor amounts of $\mathrm{Lil}\left(\mathrm{Lil} \cdot\left(\mathrm{H}_{2} \mathrm{O}\right)\right)$ and $\mathrm{Li}_{2}(\mathrm{~S} / \mathrm{Se})$ impurities can be found.

Table S4: Rietveld-refinement data of $\mathrm{Li}_{6} \mathrm{PS}_{3} \mathrm{Se}_{2}$ l and corresponding fit-residuals.

\section{$\mathrm{Li}_{6} \mathrm{PS}_{3} \mathrm{Se}_{2} \mathrm{I}$}

Lattice Parameter: 10.3010(4)

$R_{\mathrm{WP}}=7.37, R_{\mathrm{exp}}=5.39$, Goodness of fit: 1.36

Total impurity amount: $3.5 \%\left(\mathrm{LiI}, \mathrm{Li}_{2}(\mathrm{~S} / \mathrm{Se})+\mathrm{LiI}\left(\mathrm{H}_{2} \mathrm{O}\right)\right.$

\begin{tabular}{ccccccc}
\hline \multirow{2}{*}{ Atom } & $\begin{array}{c}\text { Wyckoff } \\
\text { position }\end{array}$ & \multicolumn{3}{c}{ Atomic coordinates } & \multirow{2}{*}{ Occ. } & $\boldsymbol{B}_{\text {iso }} / \AA^{\mathbf{2}}$ \\
\cline { 2 - 5 } S1 & $4 d$ & 0.25 & 0.25 & 0.25 & $0.46(4)$ & $1.4(1)$ \\
\hline Se1 & $4 d$ & 0.25 & 0.25 & 0.25 & $0.47(4)$ & $1.4(1)$ \\
\hline I1 & $4 d$ & 0.25 & 0.25 & 0.25 & $0.07(2)$ & $1.4(1)$ \\
\hline I2 & $4 a$ & 0 & 0 & 1 & $0.93(2)$ & $2.6(1)$ \\
\hline Se2 & $4 a$ & 0 & 0 & 1 & $0.07(2)$ & $2.6(1)$ \\
\hline S2 & $4 a$ & 0 & 0 & 1 & $0.00(1)$ & $2.6(1)$ \\
\hline S3 & $16 e$ & $0.1187(1)$ & $-0.1187(2)$ & $0.6187(2)$ & $0.64(1)$ & $1.87(8)$ \\
\hline Se3 & $16 e$ & $0.1187(1)$ & $-0.1187(2)$ & $0.6187(2)$ & $0.36(1)$ & $1.87(8)$ \\
\hline P & $4 b$ & 0 & 0 & 0.5 & 1 & $0.9(2)$ \\
\hline Li1 & $24 g$ & 0.25 & 0.018 & 0.75 & 0.219 & 5 \\
\hline Li2 & $48 h$ & 0.2956 & 0.0266 & 0.7044 & 0.391 & 5 \\
\hline
\end{tabular}




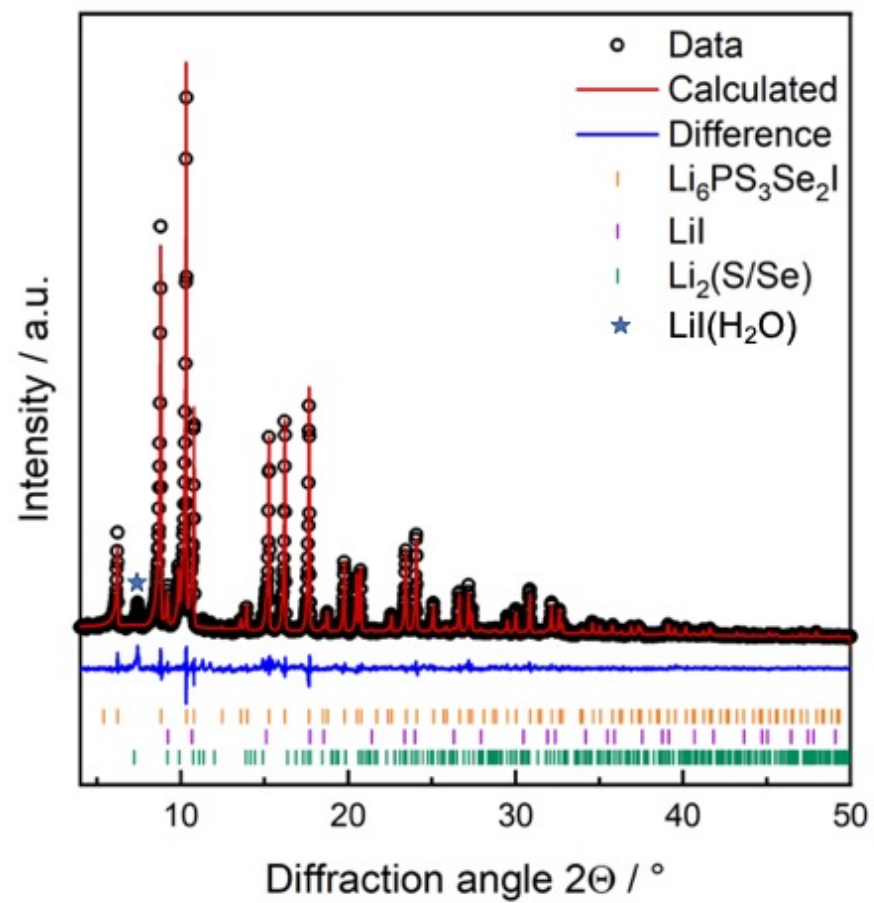

Figure S4: Diffraction pattern for $\mathrm{Li}_{6} \mathrm{PS}_{3} \mathrm{Se}_{2}$ ( (black dots), calculated structure using a Rietveld-refinement (red line) and difference curve (blue). Minor amounts of Lil $\left(\mathrm{Lil}_{(}\left(\mathrm{H}_{2} \mathrm{O}\right)\right)$ and $\mathrm{Li}_{2}(\mathrm{~S} / \mathrm{Se})$ impurities can be found.

Table S5: Rietveld-refinement data of $\mathrm{Li}_{6} \mathrm{PS}_{2} \mathrm{Se}_{3}$ l and corresponding fit-residuals.

\section{$\mathrm{Li}_{6} \mathrm{PS}_{2} \mathrm{Se}_{3} \mathrm{I}$}

Lattice Parameter: 10.3794(5)

$R_{\mathrm{WP}}=7.21, R_{\mathrm{exp}}=5.37$, Goodness of fit: 1.24

Total impurity amount: $2.8 \% \mathrm{LiI}+\mathrm{LiI}\left(\mathrm{H}_{2} \mathrm{O}\right)$

\begin{tabular}{|c|c|c|c|c|c|c|}
\hline \multirow{2}{*}{ Atom } & \multirow{2}{*}{$\begin{array}{l}\text { Wyckoff } \\
\text { position }\end{array}$} & \multicolumn{3}{|c|}{ Atomic coordinates } & \multirow{2}{*}{ Occ. } & \multirow{2}{*}{$B_{i s o} / \AA^{2}$} \\
\hline & & $x$ & $y$ & $z$ & & \\
\hline S1 & $4 d$ & 0.25 & 0.25 & 0.25 & $0.23(4)$ & $2.1(1)$ \\
\hline Se1 & $4 d$ & 0.25 & 0.25 & 0.25 & $0.72(4)$ & $2.1(1)$ \\
\hline I1 & $4 d$ & 0.25 & 0.25 & 0.25 & $0.05(2)$ & $2.1(1)$ \\
\hline I2 & $4 a$ & 0 & 0 & 1 & $0.95(2)$ & $2.5(1)$ \\
\hline $\mathrm{Se} 2$ & $4 a$ & 0 & 0 & 1 & $0.03(2)$ & $2.5(1)$ \\
\hline $\mathbf{S 2}$ & $4 a$ & 0 & 0 & 1 & $0.02(1)$ & $2.5(1)$ \\
\hline S3 & $16 e$ & $0.1200(2)$ & $-0.1200(2)$ & $0.62002)$ & $0.44(1)$ & $2.65(9)$ \\
\hline Se3 & $16 e$ & $0.1200(2)$ & $-0.1200(2)$ & $0.62002)$ & $0.56(1)$ & $2.65(9)$ \\
\hline $\mathbf{P}$ & $4 b$ & 0 & 0 & 0.5 & 1 & $2.6(2)$ \\
\hline Li1 & $24 g$ & 0.25 & 0.018 & 0.75 & 0.219 & 5 \\
\hline Li2 & $48 h$ & 0.2956 & 0.0266 & 0.7044 & 0.391 & 5 \\
\hline
\end{tabular}




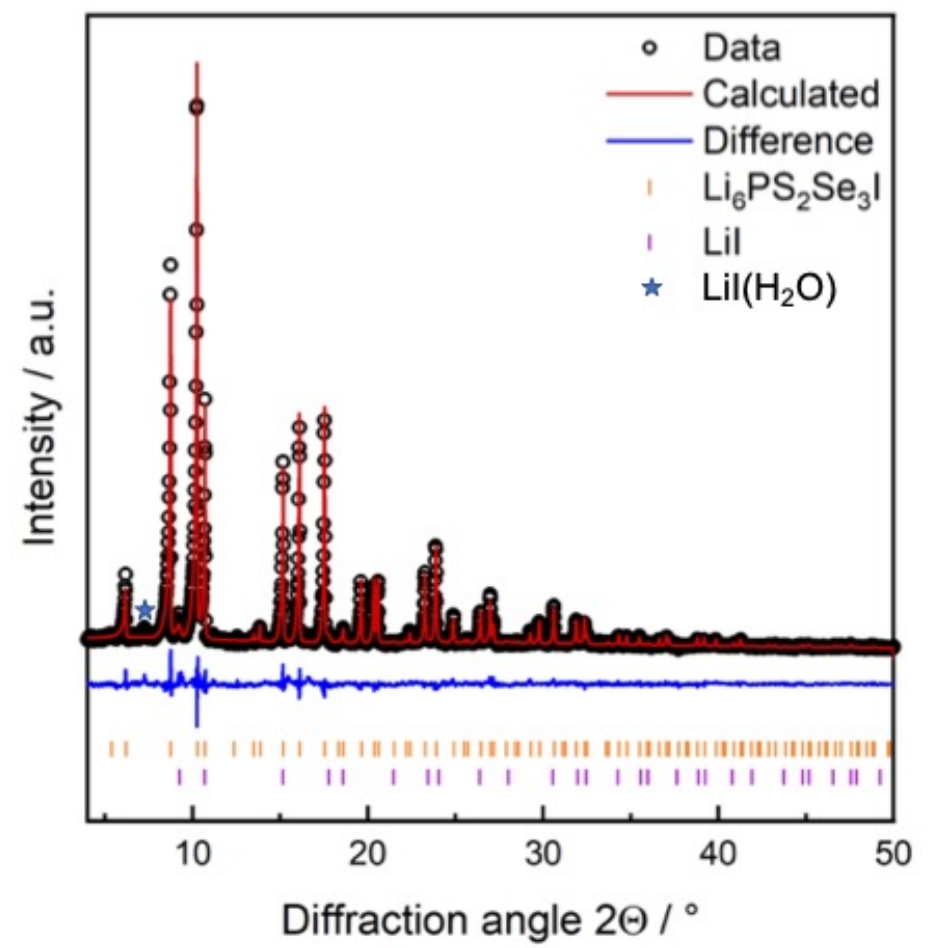

Figure S5: Diffraction pattern for $\mathrm{Li}_{6} \mathrm{PS}_{2} \mathrm{Se}_{3}$ ( (black dots), calculated structure using a Rietveld-refinement (red line) and difference curve (blue). Minor amounts of Lil $\left(\mathrm{Lil}\left(\mathrm{H}_{2} \mathrm{O}\right)\right)$ impurities can be found.

Table S6: Rietveld-refinement data of $\mathrm{Li}_{6} \mathrm{PS}_{1} \mathrm{Se}_{4}$ l and corresponding fit-residuals.

\section{$\mathrm{Li}_{6} \mathrm{PS}_{1} \mathrm{Se}_{4} \mathrm{I}$}

Lattice Parameter: 10.4625(7)

$R_{\mathrm{WP}}=6.71, R_{\mathrm{exp}}=6.07$, Goodness of fit: 1.11

Total impurity amount: $3 \%$ LiI

\begin{tabular}{ccccccc}
\hline \multirow{2}{*}{ Atom } & $\begin{array}{c}\text { Wyckoff } \\
\text { position }\end{array}$ & $\boldsymbol{x}$ & $\boldsymbol{y}$ & $\boldsymbol{z}$ & \multirow{2}{*}{ Occ. } & $\boldsymbol{B}_{\text {iso }} / \AA^{\mathbf{2}}$ \\
\cline { 2 - 6 } S1 & $4 d$ & 0.25 & 0.25 & 0.25 & $0.25(4)$ & $2.1(2)$ \\
\hline Se1 & $4 d$ & 0.25 & 0.25 & 0.25 & $0.75(4)$ & $2.1(2)$ \\
\hline I1 & $4 d$ & 0.25 & 0.25 & 0.25 & $0.07(2)$ & $2.1(2)$ \\
\hline I2 & $4 a$ & 0 & 0 & 1 & $0.93(2)$ & $2.4(1)$ \\
\hline Se2 & $4 a$ & 0 & 0 & 1 & $0.07(2)$ & $2.4(1)$ \\
\hline S2 & $4 a$ & 0 & 0 & 1 & $0.00(1)$ & $2.4(1)$ \\
\hline S3 & $16 e$ & $0.1202(2)$ & $-0.1202(2)$ & $0.6202(2)$ & $0.20(1)$ & $2.72(8)$ \\
\hline Se3 & $16 e$ & $0.1202(2)$ & $-0.1202(2)$ & $0.6202(2)$ & $0.80(1)$ & $2.72(8)$ \\
\hline P & $4 b$ & 0 & 0 & 0.5 & 1 & $3.3(3)$ \\
\hline Li1 & $24 g$ & 0.25 & 0.018 & 0.75 & 0.219 & 5 \\
\hline Li2 & $48 h$ & 0.2956 & 0.0266 & 0.7044 & 0.391 & 5 \\
\hline
\end{tabular}




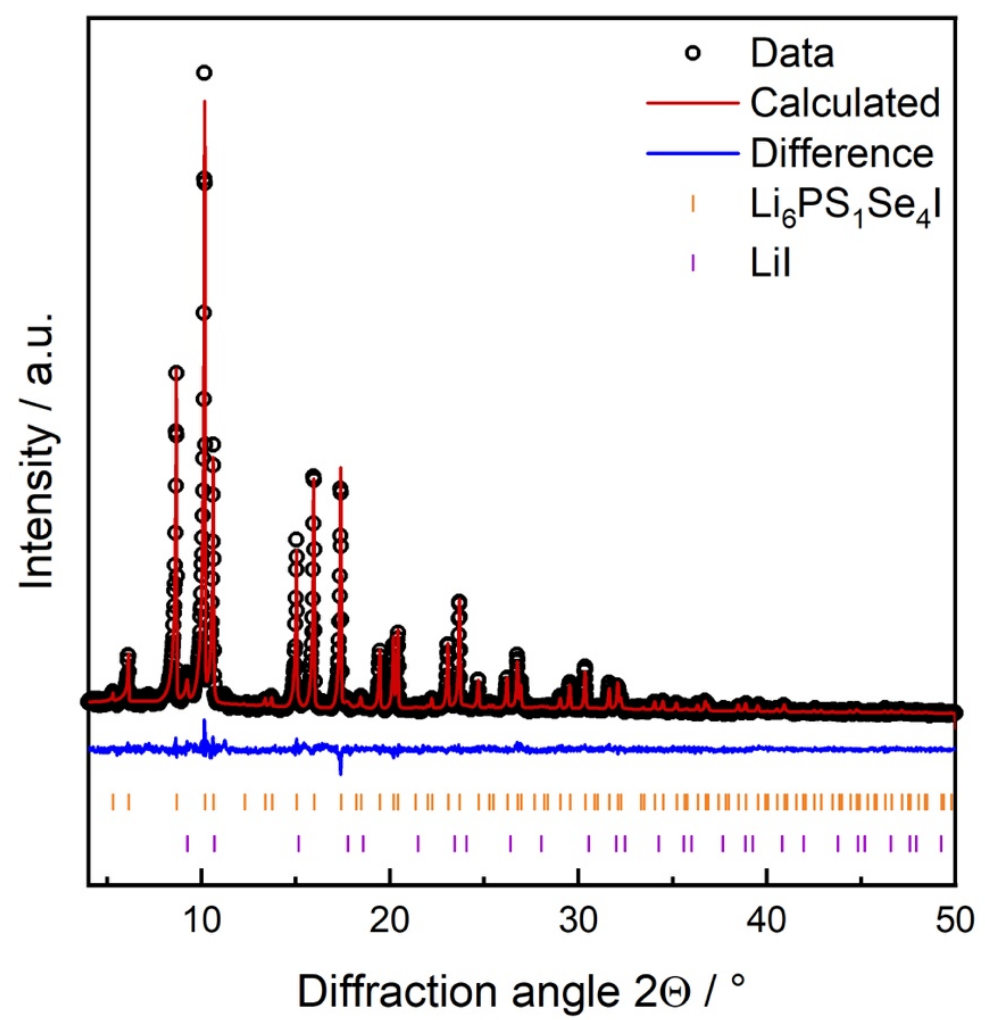

Figure S6: Diffraction pattern for $\mathrm{Li}_{6} \mathrm{PS}_{1} \mathrm{Se}_{4}$ l (black dots), calculated structure using a Rietveld-refinement (red line) and difference curve (blue). Minor amounts of Lil impurities can be found.

Table S7: Rietveld-refinement data of $\mathrm{Li}_{6} \mathrm{PSe}_{5} \mathrm{l}$ and corresponding fit-residuals.

\section{$\mathrm{Li}_{6} \mathrm{PSe}_{5} \mathrm{I}$}

Lattice Parameter: $10.5515(8)$

$R_{\mathrm{WP}}=6.74, R_{\mathrm{exp}}=6.66$, Goodness of fit: 1.01

Total impurity amount: $3 \%$ LiI

\begin{tabular}{|c|c|c|c|c|c|c|}
\hline \multirow{2}{*}{ Atom } & \multirow{2}{*}{$\begin{array}{l}\text { Wyckoff } \\
\text { position }\end{array}$} & \multicolumn{3}{|c|}{ Atomic coordinates } & \multirow{2}{*}{ Occ. } & \multirow{2}{*}{$B_{i s o} / \AA^{2}$} \\
\hline & & $x$ & $y$ & $z$ & & \\
\hline Se1 & $4 d$ & 0.25 & 0.25 & 0.25 & $0.97(4)$ & $1.9(2)$ \\
\hline I1 & $4 d$ & 0.25 & 0.25 & 0.25 & $0.03(2)$ & $1.9(2)$ \\
\hline I2 & $4 a$ & 0 & 0 & 1 & $0.97(4)$ & $3.1(2)$ \\
\hline Se2 & $4 a$ & 0 & 0 & 1 & $0.03(2)$ & $3.1(2)$ \\
\hline Se3 & $16 e$ & $0.1205(2)$ & $-0.1205(2)$ & $0.6205(2)$ & 1 & $2.45(5)$ \\
\hline $\mathbf{P}$ & $4 b$ & 0 & 0 & 0.5 & 1 & $1.6(2)$ \\
\hline Li1 & $24 g$ & 0.25 & 0.018 & 0.75 & 0.219 & 5 \\
\hline Li2 & $48 h$ & 0.2956 & 0.0266 & 0.7044 & 0.391 & 5 \\
\hline
\end{tabular}




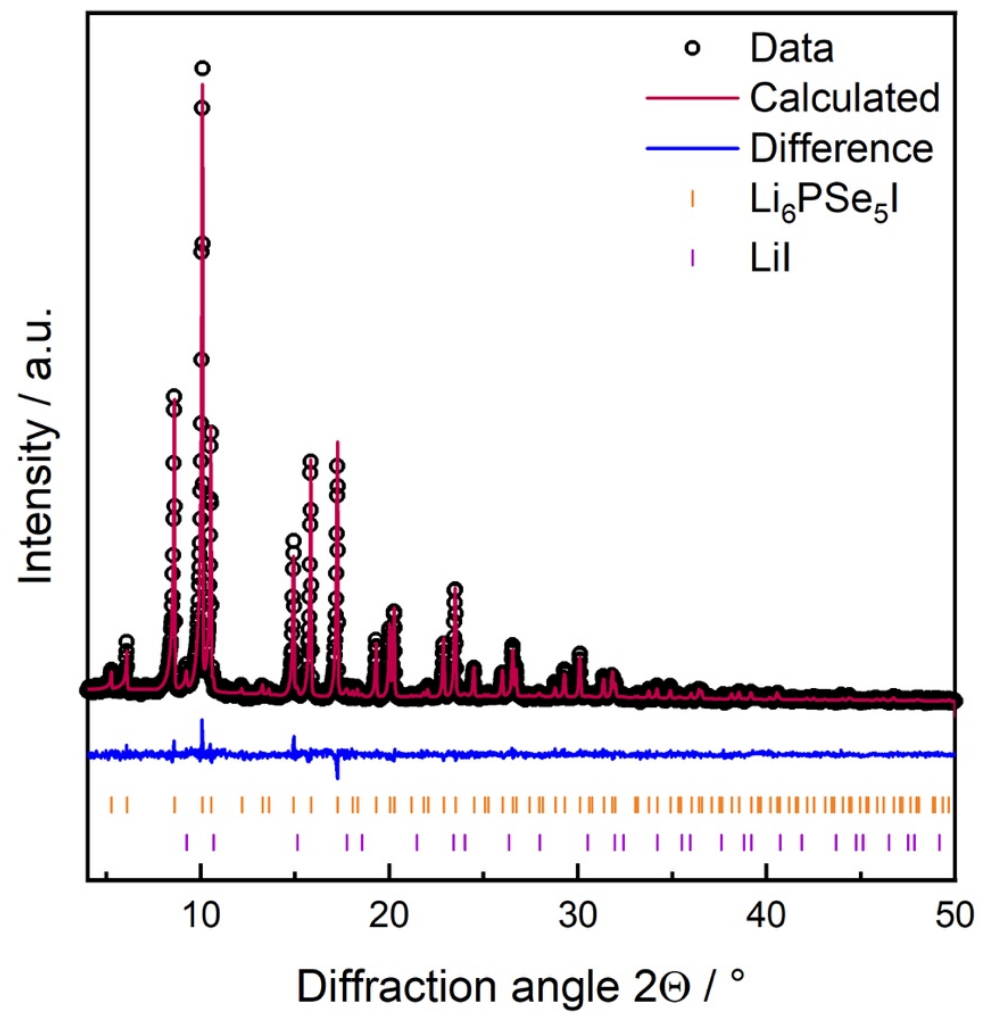

Figure S7: Diffraction pattern for $\mathrm{Li}_{6} P \mathrm{PS}_{5}$ l (black dots), calculated structure using a Rietveld-refinement (red line) and difference curve (blue). Minor amounts of Lil impurities can be found.

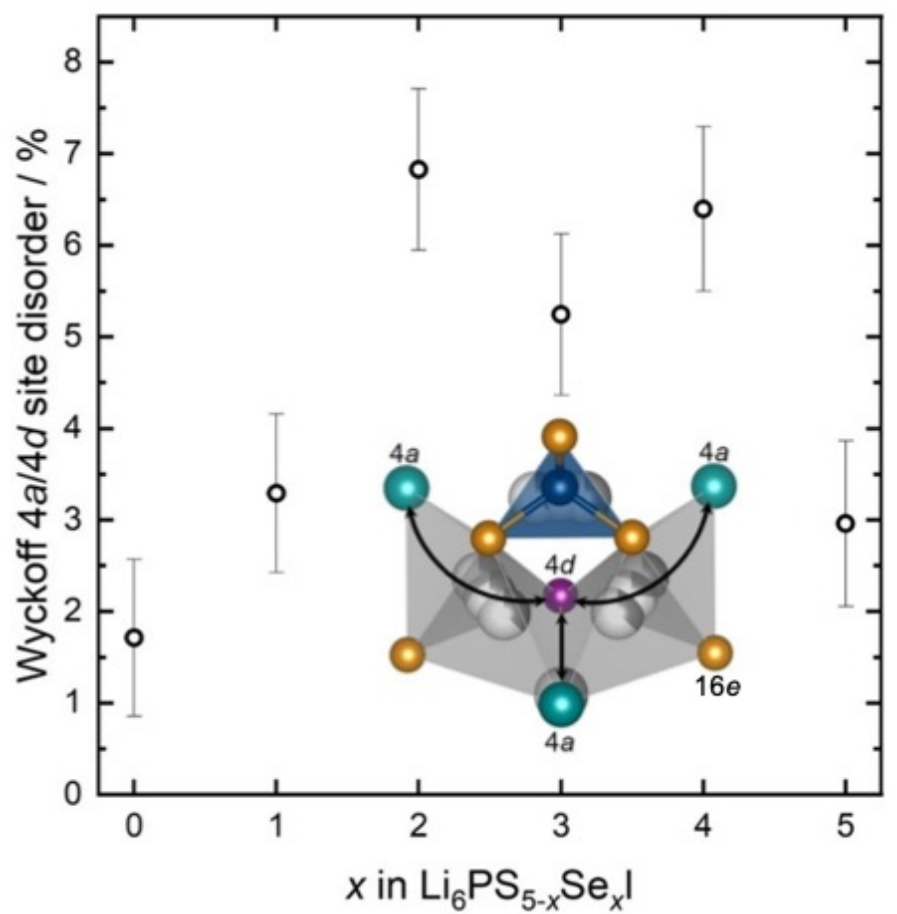

Figure S8: Site-disorder between the Wyckoff $4 d$ and $4 a$ site as obtained from Rietveld-Refinements. The large amount of necessary constraints makes a Rietveld-Refinement difficult and the here shown values represent a general trend, rather than absolute values. No direct correlation between the site-disorder and transport properties could be observed. 